\title{
Causes of arachnoid cyst development and expansion
}

\author{
Luca Basaldella, M.D., ${ }^{1}$ Enrico Orvieto, M.D, ${ }^{2}$ Angelo Paolo Dei Tos, M.D., ${ }^{2}$ \\ Mila Della Barbera, M.D. ${ }^{3}$ Marialuisa Valente, M.D., ${ }^{3}$ \\ and Pierluigi Longatti, M.D. ${ }^{1}$
}

${ }^{1}$ Department of Neurosurgery, Treviso Regional Hospital, University of Padova; ${ }^{2}$ Department of Pathology, Treviso Regional Hospital; and ${ }^{3}$ Department of Pathology, University of Padova, Italy

\begin{abstract}
$\checkmark$ Arachnoid cysts are frequent incidental findings on neuroimaging studies and in clinical practice. Theories of their origin, still matter for debate, compose four categories: 1) a ball-valve mechanism; 2) an osmotic gradient between the intra- and extracystic medium; 3) primary malformation of the arachnoid membrane or cerebral lobe agenesis; and 4) fluid hypersecretion by the lining cells of the cyst wall. The cause of cyst enlargement is also debatable, although there is strong controversial evidence supporting the last two theories rather than the former. Brain water homeostasis and its regulatory pathways are weakly understood at the molecular level. In this brief report the authors attempt to add new insights into the pathogenesis of arachnoid cysts by considering aquaporin expression in the cyst wall and discuss possible future research directions and molecular targets.
\end{abstract}

KEY WORDS • arachnoid cyst • hydrocephalus • aquaporin

A RACHNOID cysts are intraarachnoid sacs containing CSF-like fluid that do not communicate with the ventricular system. They account for approximately $1 \%$ of all intracranial masses, with 50 to $60 \%$ occurring in the middle cranial fossa. Most arise as developmental anomalies. ${ }^{47,14,15,17}$ A small number are associated with neoplasms or can occur as complications following leptomeningitis, hemorrhage, or surgery. ${ }^{5}$ They also occur within the spinal canal, ${ }^{6}$ with cysts or diverticula located subdurally or in the epidural space. ${ }^{4,5}$ Spinal arachnoid cysts are commonly located dorsal to the cord in the thoracic region. Intradural spinal arachnoid cysts are caused by a congenital deficiency within the arachnoid or are the result of adhesions due to previous infection or trauma. ${ }^{5}$ Ultrastructural examination has shown that the cyst wall is formed from a splitting of the arachnoid membrane, with an inner and outer leaflet surrounding the cyst cavity. ${ }^{13-15}$ The origin of arachnoid cysts is still a matter of debate. Despite the numerous scientific theories, none has been proven yet. In summary, there are four proposed theories regarding the cause of these cysts: 1) a ball-valve mechanism (that is, a possible anatomical communication between the cyst and the subarachnoid space that can act as a one-way valve mechanism responsible for cyst enlargement);3,9,16 2) an osmotic gradient between the intra- and extracystic medium responsible for a gradientdriven fluid transport (note that this theory lacks support given the great compositional similarity between CSF and the cyst's fluid content);17 3) a malformation (that is, trapped fluid content in cerebral lobe agenesis); $; 4,6$ and

Abbreviations used in this paper: $\mathrm{AQP}=$ aquaporin; $\mathrm{CSF}=$ cerebrospinal fluid; NPA = asparagine-proline-alanine.
4) hypersecretive fluid production by cells lining the luminal cyst's wall. $6,8,13-15,17,18$

Aquaporins act as putative water channels that have offered new insights into the water balance preservation of many anatomical regions in humans, in animals, and in plants and other microorganisms. ${ }^{1}$ They compose a family of 13 recognized members, numbered from 0 to $12 . .^{1,2,19}$ They are ubiquitously distributed but preferentially focused in the epithelial lining cells of tissues specialized for water secretion and absorption such as renal tubules and collecting ducts, ${ }^{1}$ the blood-brain barrier, and the CSF-blood barrier. ${ }^{2}$ In the central nervous system mainly three AQPs have been identified: AQP1 in the choroid plexus, ${ }^{10,12}$ AQP4 in the astrocytic end-feet of the blood-brain barrier, ${ }^{2}$ and AQP9, which was first described in tanycytes and glucose-sensitive neurons. ${ }^{1,2}$ The structural organization of AQP1 reflects a general feature of the AQP family (Fig. 1): ${ }^{2}$ conformational analysis of the amino acid sequence suggests a six transmembrane-spanning topography for each AQP molecule with cytosolic amino and carboxy termini with three extracellular and two intracellular loops, respectively. The amino acid sequence shows a strong similarity between the two halves of the molecule, indicating a probable evolutionary intergenic duplication. A highly conserved three amino acid motif, NPA, is present in the B and E loops of nearly all AQPs. The NPA motifs are inserted into the membrane. The intracellular B loop and the extracellular E loop fold into the membrane and interact with one another, forming a sort of hourglass characterized by wide external openings to the channel with a narrow central constriction where the NPA motifs interact and form the functional permeable pore. Taking into account the hyper- 


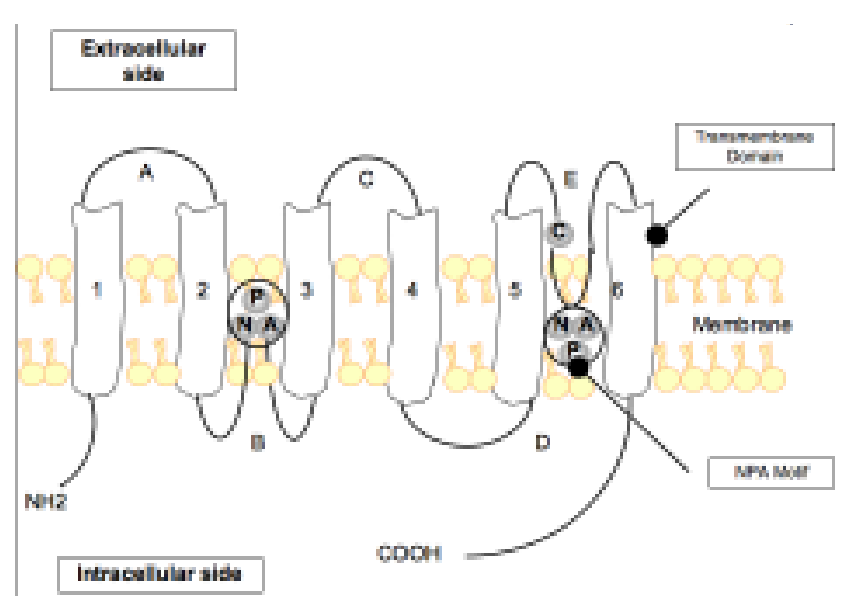

FIG. 1. Schema illustrating a model of AQP in the cell membrane. The subunit of the tetrameric water channel is formed from six transmembrane domains. Transmembrane hydrophobic $\alpha$ helices are numbered from 1 to 6 . The NPA motif is a highly conserved amino acid sequence inserted into the membrane constant for all AQPs in B and D domains. They interact, forming the functional hydrophilic pore.

secretion theory, we tested surgical specimens of normal arachnoid membrane, arachnoid cysts, and arachnoid villi for the expression of AQP1, the second member of the AQP family. We have previously studied APQ1 and found it abundantly expressed in the choroid plexus (Fig. 2), ${ }^{10}$ choroid plexus tumors (Fig. 3) ${ }^{12}$ associated with hypersecretive hydrocephalus, and cystic hemangioblastomas. ${ }^{11}$ The aim of this preliminary investigation was to describe the anatomical distribution of AQP1 in arachnoidal specimens in an attempt to find new data for the pathophysiological interpretation of arachnoid cyst formation and development.

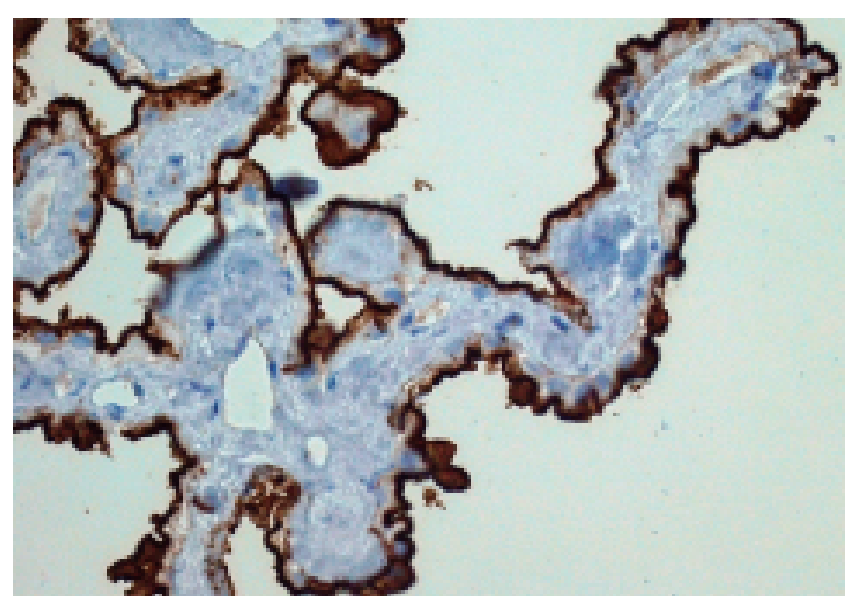

FIG. 2. Photomicrograph depicting normal choroid plexus with papillary projections. Immunostaining with AQP1 epitope (clone ab9566, Abcam) reveals cuboid cells expressing AQP1 at the highest degree in correspondence with the microvilli of the apical portion. Note the AQP1 expression all along the CSF-submerged surface of the choroid plexus as an uninterrupted line. Original magnification $\times 200$.

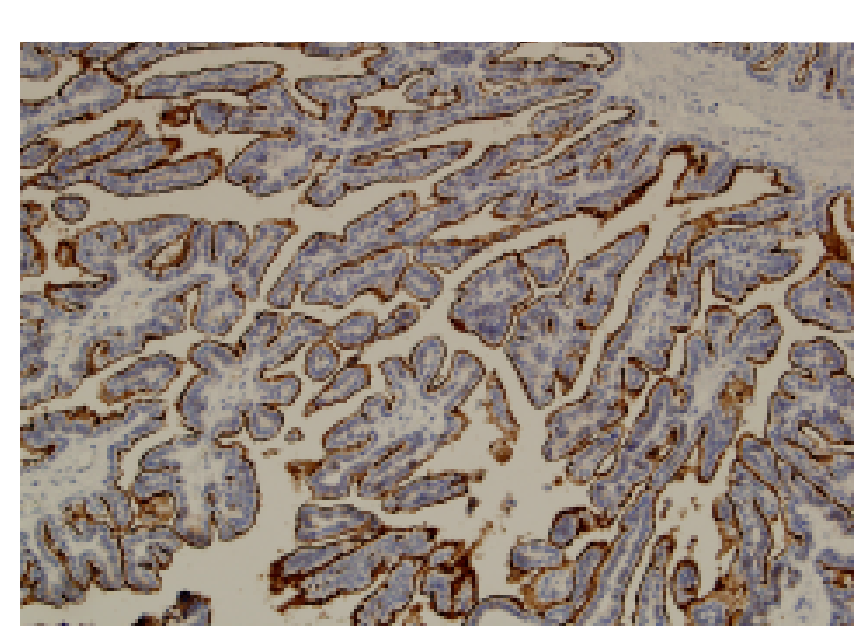

FIG. 3. Photomicrograph demonstrating hyperexpression of AQP1 in a choroid plexus papilloma with AQP1 epitope (clone ab9566, Abcam) immunostaining. Original magnification $\times 200$.

\section{Materials and Methods}

We analyzed six specimens obtained during neurosurgical procedures: two normal arachnoid layers, two specimens of arachnoid cysts, and two arachnoid villi. Tissue specimens were fixed in $10 \%$ formaldehyde $/ 0.9 \% \mathrm{NaCl}$ and embedded in paraffin wax after 24 hours. Part of each specimen was then stained with $\mathrm{H} \& \mathrm{E}$ for diagnostic control. Coronal sections (5 $\mu \mathrm{m}$ thick) were collected on polylysine-coated slides and immunostained with a monoclonal antibody against the intracellular C-terminal AQP1 epitope (clone ab9566 1/22, dilution 1:100, Abcam). Immunohistochemical stainings were performed using a sensitive polymer-based detection system (Envision Plus, DakoCytomation). Heat-induced antigen retrieval was performed by incubating the slides for 40 minutes at $98^{\circ} \mathrm{C}$ in a water bath and using $7.0 \mathrm{pH}$ citrate buffer. All immunostainings were performed on an automated immunostainer (Dako Autostainer, DakoCytomation).

\section{Results}

No AQP1 immunoreactivity was found in the specimen of the normal arachnoid layer, in the arachnoid villus, or in the cells lining the cystic wall (Fig. 4 and 5).

\section{Discussion}

Congenital arachnoid cysts are developmental anomalies and contain clear CSF-like fluid. Arachnoid cysts most likely originate from a minor aberration in the development of the arachnoid that leads to splitting or duplication of the membrane ${ }^{4,6,7}$ It has also been postulated that the cyst develops from a defect in condensation of the mesenchyme or from abnormalities of CSF flow. $13,14,15,17,20$ The association of other developmental abnormalities of the brain, such as heterotopias, lent support to this developmental theory. In the 208 reported cases of arachnoid cysts analyzed by Rengachary and colleagues, ${ }^{14,15}$ they found that the structural features of the arachnoid cyst wall that distinguish it from the normal arachnoid membrane were as follows: 1) splitting of the arachnoid membrane at the margin of the cyst; 2) a thick layer of collagen 


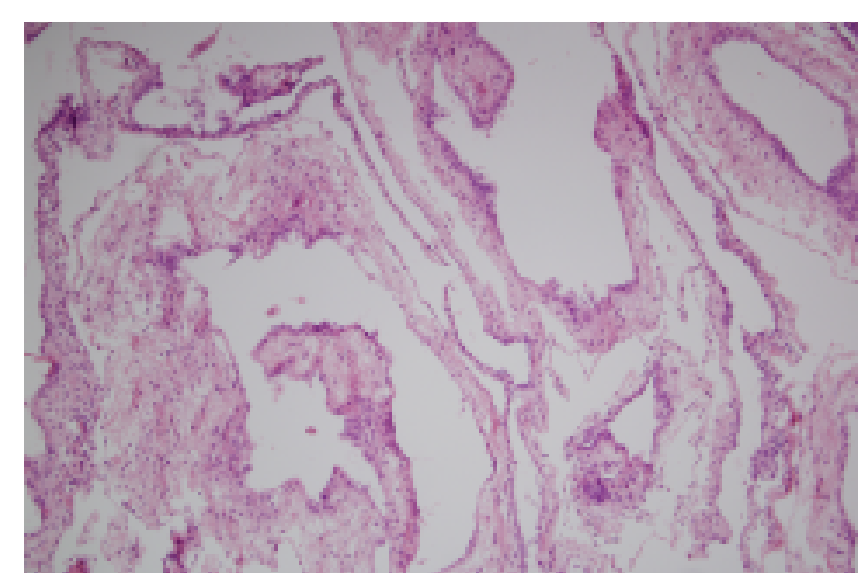

FIG. 4. Photomicrograph revealing a normal arachnoid membrane. H \& E, original magnification $\times 200$.

in the cyst wall; 3) the absence of traversing trabecular processes within the cyst; and 4) the presence of hyperplastic arachnoid cells in the cyst wall.

In a study of five cases, Miyagami and Tsubokawa ${ }^{13}$ reported that the structure of the arachnoid cyst wall was similar to that of the normal arachnoid membrane and that the inner surface of the arachnoid cyst wall was formed of one or several layers of arachnoid cells with slender processes, which contained large extracellular spaces but no microvilli. These cysts appeared to be truly intraarachnoid in location and to be formed by splitting or duplication of the arachnoid membrane..$^{14,15}$ In a study of nine cases, Schachenmayr and Friede ${ }^{17}$ found that the dominant phenomenon in the cyst wall was an absence of the normal trabeculation of the subarachnoid space, the trabeculae being replaced by tightly packed collagen fibrils and a few scattered cells in between. They found no evidence of a tight sealing of the extracellular spaces in the cyst wall.

A number of interesting theories have been proposed to explain the expansion of arachnoid cysts. According to the

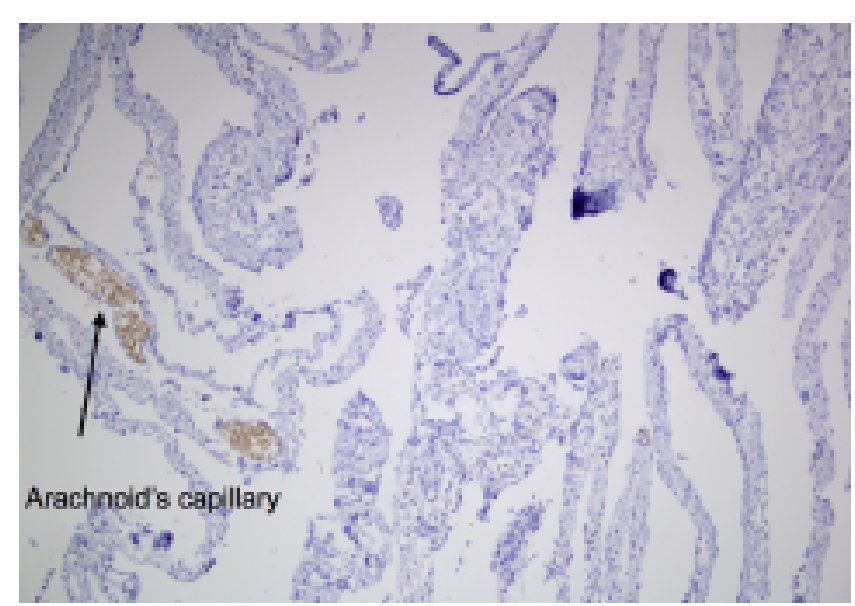

FIG. 5. Photomicrograph showing the results of immunohistochemical staining with AQP1 epitope (clone ab9566, Abcam) for AQP1 in an arachnoid cyst membrane. It is possible to depict AQP1 expression in red blood cells of an arachnoid capillary but not in the cells lining the cyst wall. Original magnification $\times 100$.

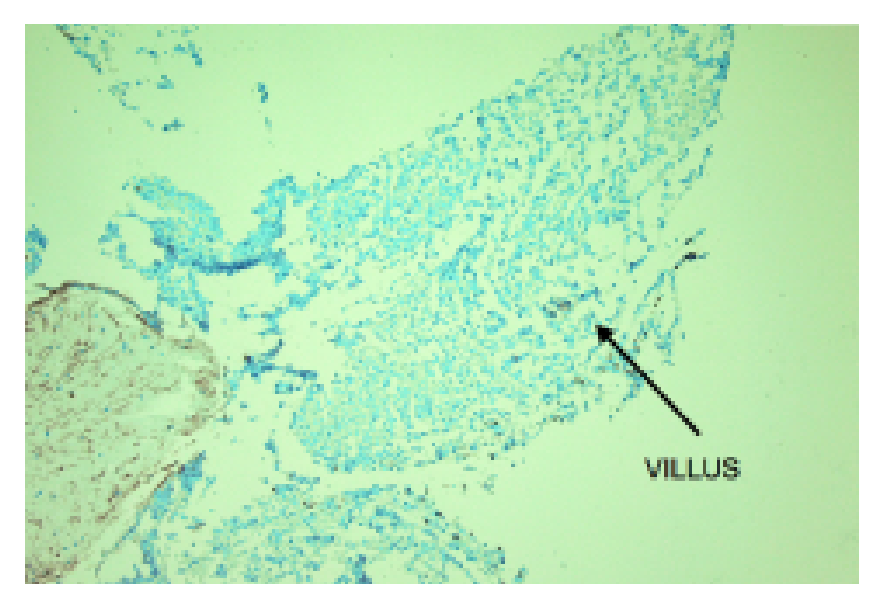

FIG. 6. Photomicrograph revealing immunohistochemical staining with AQP1 epitope (clone ab9566, Abcam) for AQP1 in an arachnoid villus. Note the complete absence of AQP1 expression in this anatomical structure. Original magnification $\times 200$.

ball-valve hypothesis, an anatomical fissure of the cyst wall functionally acts as a one-way valve, allowing free entrance of CSF but preventing its exit into the subarachnoid space. One-way pulsatile movement of CSF has been demonstrated by cine-mode magnetic resonance imaging studies and confirmed at endoscopic operations by inspecting the inside of the cyst wall. ${ }^{16}$ The theory of an osmotic gradient between cystic contents and CSF lacks support given that the cystic content is similar in composition to CSF. ${ }^{17}$ There has been no evidence of either a tight sealing of the extracellular spaces in the cyst wall or an active transcellular fluid movement in some studies. ${ }^{14,15}$

Fluid production by the cells lining the cyst wall is another aspect that has been considered. There is morphological and ultrastructural evidence to support the secretory nature of the cyst wall. Ultrastructurally, the cyst lining cells demonstrate the presence of microvilli on the luminal surface and cytoplasmic vesicles that are consistent with fluid secretion. ${ }^{8}$ Moreover, enzyme immunocytochemistry demonstrated $\mathrm{Na}-\mathrm{K}$ adenosine triphosphatase in the plasma membranes lining the cavity at the luminal side and near the intercellular clefts at the basolateral side, a structural organization consistent with fluid transport toward the lumen. ${ }^{6}$ The argument against continuous secretion lay in the fact that cysts often remain static in size and sometimes disappear, thus demonstrating that secretion is neither universal nor likely the only mechanism involved. Schuhmann and colleagues ${ }^{18}$ have described choroid plexus ectopia inside a growing arachnoid cyst. Our results seem to put the hypersecretion theory into the right perspective. Considering our previous data, we tested AQP1, which seems to be the most realistic member expressed in this kind of disorder. Given the technical limits of immunohistochemistry, it is impossible to preclude the expression of other members of the AQP family at the subcellular level. There was no staining for AQP1 in the arachnoid villi (Fig. 6), where CSF absorption occurs, thus offering more evidence for the mechanical theory of CSF pressure gradient absorption and for translation to the ball-valve mechanism of cyst development. 


\section{References}

1. Agre P, Bonhivers M, Borgnia MJ: The aquaporins, blueprints for cellular plumbing systems. J Biol Chem 273:14659-14662, 1998

2. Borgnia M, Nielsen S, Engel A, Agre P: Cellular and molecular biology of the aquaporin water channels. Annu Rev Biochem 68:425-458, 1999

3. Brooks ML, Jolesz FA, Patz S: MRI of pulsatile CSF motion within arachnoid cysts. Magn Reson Imaging 6:575-584, 1988

4. Catala M, Poirier J: [Arachnoid cysts: histologic, embryologic and physiopathologic review.] Rev Neurol 154:489-501, 1998 (Fr)

5. Choi JU, Kim DS: Pathogenesis of arachnoid cyst: congenital or traumatic? Pediatr Neurosurg 29:260-266, 1998

6. Clemenceau S, Carpentier A: [Intracranial arachnoid cysts. A review.] Rev Neurol 155:604-608, 1999 (Fr)

7. Garcia Santos JM, Martinez-Lage J, Gilabert Ubeda A, Capel Aleman A, Climent Oltra V: Arachnoid cysts of the middle cranial fossa: a consideration of their origins based on imaging. Neuroradiology 35:355-358, 1993

8. Go KG, Houthoff HJ, Blaauw EH, Havinga P, Hartsuiker J: Arachnoid cysts of the sylvian fissure. Evidence of fluid secretion. J Neurosurg 60:803-813, 198

9. Hoffmann KT, Hosten N, Meyer BU, Roricht S, Sprung C, Oellinger $\mathrm{J}$, et al: CSF flow studies of intracranial cysts and cyst-like lesions achieved using reversed fast imaging with steady-state precession MR sequences. AJNR Am J Neuroradiol 21:493-502, 2000

10. Longatti PL, Basaldella L, Orvieto E, Fiorindi A, Carteri A: Choroid plexus and aquaporin-1: a novel explanation of cerebrospinal fluid production. Pediatr Neurosurg 40:277-283, 2004

11. Longatti P, Basaldella L, Orvieto E, Tos AP, Martinuzzi A: Aquaporin 1 expression in cystic hemangioblastomas. Neurosci Lett 392:178-180, 2006
12. Longatti P, Basaldella L, Orvieto E, Tos AP, Martinuzzi A: Aquaporin(s) expression in choroid plexus tumors. Pediatr Neurosurg 42:228-233, 2006

13. Miyagami M, Tsubokawa T: Histological and ultrastructural findings of benign intracranial cysts. Noshuyo Byori 10: 151-160, 1993

14. Rengachary SS, Watanabe I: Ultrastructure and pathogenesis of intracranial arachnoid cysts. J Neuropathol Exp Neurol 40: 61-83, 1981

15. Rengachary SS, Watanabe I, Brackett CE: Pathogenesis of intracranial arachnoid cysts. Surg Neurol 9:139-144, 1978

16. Santamarta D, Aguas J, Ferrer E: The natural history of arachnoid cysts: endoscopic and cine-mode MRI evidence of a slitvalve mechanism. Minim Invasive Neurosurg 38:133-137, 1995

17. Schachenmayr W, Friede RL: Fine structure of arachnoid cysts J Neuropathol Exp Neurol 38:434-446, 1979

18. Schuhmann MU, Tatagiba M, Hader C, Brandis A, Samii M: Ectopic choroid plexus within a juvenile arachnoid cyst of the cerebellopontine angle: cause of cyst formation or reason of cyst growth. Pediatr Neurosurg 32:73-76, 2000

19. Venero JL, Vizuete ML, Machado A, Cano J: Aquaporins in the central nervous system Prog Neurobiol 63:321-336, 2001

20. Wester K: Peculiarities of intracranial arachnoid cysts: location, sidedness, and sex distribution in 126 consecutive patients. Neurosurgery 45:775-779, 1999

Manuscript submitted November 16, 2006.

Accepted January 8, 2007.

Address reprint requests to: Luca Basaldella, M.D., Divisione Clinicizzata di Neurochirurgia, Ospedale Regionale di Treviso, Ca'Foncello, Facoltà di Medicina e Chirurgia-Università degli Studi di Padova, Piazzale Ospedale Civile 1, 31100, Treviso, Italia. email: luca.basaldella@mac.com. 